\title{
Motor learning by cross education in Hemi-Parkinson's disease: Case study of the effects of virtual mirrored sensory feedback
}

Ori Ossmy ${ }^{1}$, Lihi Mansano², Silvi Frenkel-Toledo ${ }^{3,2}$, Evgeny Kagan ${ }^{4}$, Shiri Koren ${ }^{6}$, Roee Gilron ${ }^{4,6}$, Daniel Reznik ${ }^{4,6}$, Nachum Soroker ${ }^{2,5}$, Roy Mukamel ${ }^{4,6}$

${ }^{1}$ Department of Psychology, New York University, New York, NY, United States.

2 Department of Neurological Rehabilitation, Loewenstein Hospital, Ra'anana, Israel.

3 Department of Physiotherapy, Faculty of Health Sciences, Ariel University, Ariel, Israel.

${ }^{4}$ Sagol School of Neuroscience, Tel-Aviv University, Tel-Aviv, Israel.

5 Sackler Faculty of Medicine, Tel-Aviv University, Tel Aviv, Israel.

6 School of Psychological Sciences, Tel-Aviv University, Tel-Aviv, Israel.

\section{Corresponding Author}

Roy Mukamel

School of Psychological Sciences \& Sagol School of Neuroscience

Tel-Aviv University, Tel-Aviv, 69978, Israel

+972-3-6407246

Email: rmukamel@tau.ac.il 


\section{Abstract}

Cross education is a phenomenon in which motor training of one hand induces motor learning in the other hand. We have recently shown in healthy subjects that the effect of cross-education is significantly augmented by provision of real-time manipulated bi-modal (visual and kinesthetic) sensory feedback, creating an illusory sensation of voluntary training with the other hand. Here we tested whether this training method may be applicable also in pathological conditions affecting one side of the body. We present here data showing behavioral gain accompanied by functional magnetic resonance imaging dynamics following training with this setup in the case of patient LA, a young man with significant unilateral upper-limb dysfunction stemming from hemi-Parkinson's disease. Following two weeks of daily sessions in which he intensively trained the non-affected upper limb, he showed improvement in motor capacity of the affected limb, accompanied by enhanced activation in the pre-frontal cortex and a widespread increase in functional coupling in the brain. Results from the current case study suggest that combining cross-education with manipulated sensory input may also have beneficial effects in clinical conditions.

Keywords: Cross-Education; Upper-limb hemiparesis; Mirror sensory feedback; Proprioceptive feedback; 


\section{Introduction}

It has been known for over a century that physical training with one limb can result in substantial performance gains also in the untrained limb - a phenomenon known as Cross-Education (CE $)^{1-3}$. CE was used in the clinical realm mainly in the context of immobilization therapy. During immobilization of a limb, there is significant loss of muscle mass. Physical training of the contralateral limb has been shown to prevent, or slow down this process ${ }^{4,5}$. CE also supports transfer of grip-precision learning from the unaffected to the affected hand in patients with unilateral upper-limb dysfunction following stroke ${ }^{6}$.

We have recently developed a unique CE setup which incorporates Virtual Reality (VR) in the form of manipulated visual and proprioceptive feedback, creating an illusory perception of voluntary movement in the non-trained upper limb $(\mathrm{UL})^{7,8}$. Healthy subjects that trained with this setup showed high performance outcomes in the hand that was not under direct voluntary control. The augmented effect of CE in a VR setup points to the importance of sensory feedback as a source of afferent information used for evaluation and online correction of executed movement, as well as for motor learning ${ }^{9}$. One form of visual input used in clinical practice is mirror visual feedback (MVF), where movement of the non-affected UL is viewed in a mid-sagittal mirror, creating an illusory percept of movement in the affected UL. Such addition of MVF training to the standard clinical rehabilitation process has been shown to have beneficial efects ${ }^{10-13}$. Importantly, using sensory input to improve motor performance is not limited to vision ${ }^{9}$. In proprioceptive training, the affected limb is typically strapped to a device that is controlled either by a computer ${ }^{14,15}$ or by the subject's other limb ${ }^{7}$. In either case, the limb moves passively and the subject receives proprioceptive input similar to what he would have received during voluntary controlled movement. Proprioceptive training was shown to facilitate rehabilitation of hemiparetic stroke patients ${ }^{16-18}$ and also Parkinsonian patients ${ }^{19}$.

Parkinson's disease is a prevalent cause of motor disability, mainly but not exclusively among elderly people, which often has its initial presentation in the form of Hemi-Parkinson's disease unilateral impairment of motor function, with one or more of the following manifestations: muscle rigidity, tremor, hypokinesia and bradykinesia, impaired force regulation, impaired motor learning and difficulty alternating between different motor acts. The condition may remain in unilateral or largely asymmetric form for long periods of time 20,21 . Here we tested the translation of our findings in healthy subjects to the realm of clinical practice. Specifically, we examined in a highly motivated young man whose right hand was severely affected by hemi-Parkinson's disease, the effectiveness of training the non-affected left hand with our novel CE setup. Additionally, by using whole-brain functional magnetic resonance imaging (fMRI) we probed potential neural mechanisms underlying the training effects.

\section{Methods}

$\underline{\text { Patient }}$

LA is a 46-years old right handed male with 18 years of formal schooling, working as a manager in energy projects. He is married, father of 3 sons and has no family history of neurological disorders. In early 2012 he started to experience difficulties using his dominant right hand during execution of fine motor skills, with a noticed change in handwriting. His neurological examination at that time and an MRI scan done somewhat later didn't reveal any pathology. However, a scan detecting dopamine transporters (DaT Scan) showed depletion of striatal dopaminergic neurons suggestive of Parkinson's disease. Treatment with Amantadine, Rasagiline and Pramipexole did not ameliorate the hand function and eventually these medications were discontinued. Currently LA shows serious slowness and rapid 
fatigue in repetitive movement of the right hand with slight focal hypertonicity. In addition he suffers from a restless right leg during intense activity calming at rest, for which treatment with Biperiden has been tried. LA keeps a daily aerobic exercise routine and receives two physiotherapy sessions per week. He also performs a set of daily exercises at home aimed to improve control of fine movement in the affected right hand. The exercises included copying a 5 sentence paragraph, counting the number of finger taps he performs in a minute, drawing a star contour next to a sample star viewed in a mirror, and drawing a line from the starting point of a maze to its end point without touching the outline. LA provided written informed consent to participate in the study which conformed to the guidelines approved by the Ethics committee at Tel-Aviv University and the Helsinki committee at the Beit Loewenstein Rehabilitation Center.

\section{Training setup}

The training setup designed for $L A$ is based on our earlier research with healthy subjects ${ }^{8}$. LA was trained while sitting in a chair with the two upper limbs in a forward position and the hand palms positioned in a specialized motion control apparatus (see Fig. 1a). The device restricts voluntary movement of the right hand fingers and only left hand finger movements activate the motors ${ }^{7,8}$. Thus, voluntary left (non-affected) hand finger movement results in passive yoking of the corresponding fingers of the right (affected) hand. In addition, LA wore a VR headset ${ }^{8}$ that prevented visual input of his real hands and the device and provided visual feedback of virtual hands instead. LA also wore motion-sensing MR-compatible gloves ${ }^{8}$ that allow online monitoring of individual finger flexion in each hand. A head-mounted specialized 3D camera ${ }^{8}$ provided online visual feedback of the real environment. Together, these devices allowed detection of LA's real hand movements and translating them by customized software to virtual hand movements presented on the headset screen. The virtual hands were embedded in a specific location in space and were presented only when LA looked down towards the natural position of his hands.

\section{Experiment design}

The experiment started following two consecutive days in which LA's manual function was evaluated using a battery of clinical tests. The experiment comprised of two parts (See Fig. 1b) - a standard training part (10 days in which he continued doing his daily exercises) and an intervention part ( 9 days of training with the experimental setup), with an intervening period of 6 days in which he did not practice at all. During the standard training, LA conducted his regular daily exercises (see Methods/Patient above) at 8AM, 5 days per week, during 2 consecutive weeks. In the first and last day of the standard training, LA underwent clinical performance evaluation (see Behavior: performance evaluation). A week after the standard training ended, LA underwent another clinical evaluation to examine retention levels. During the intervention period (See Fig. 1C), LA trained to execute rapid sequences of finger movements using our novel setup on a daily basis. Fig. $1 \mathrm{~b}$ demonstrates the timeline of a single intervention day. In an initial evaluation stage, LA was instructed to perform a unimanual 5-digit finger sequence movement: 3-1-4-2-3 (See Fig. 1), repeatedly as accurately and rapidly as possible with the affected hand. Performance level was measured as the number of correct sequences performed in 60 seconds. Following the pre-training evaluation stage, a "Start" sound was played for 2 seconds cueing LA to the upcoming training stage in which he performed the sequence of finger movements with his non-affected hand in the special training setup in a self-paced manner (see training setup). Training blocks lasted 10 minutes and were followed by 10 minutes of rest. Each block consisted of four trials and each trial consisted of 2-minute training, followed by 30 seconds of a yellow 


\section{Training setup}

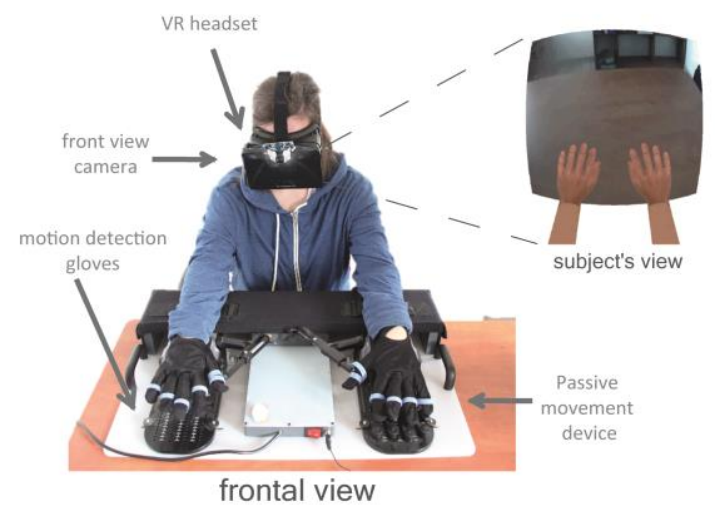

b

\section{Single intervention day}

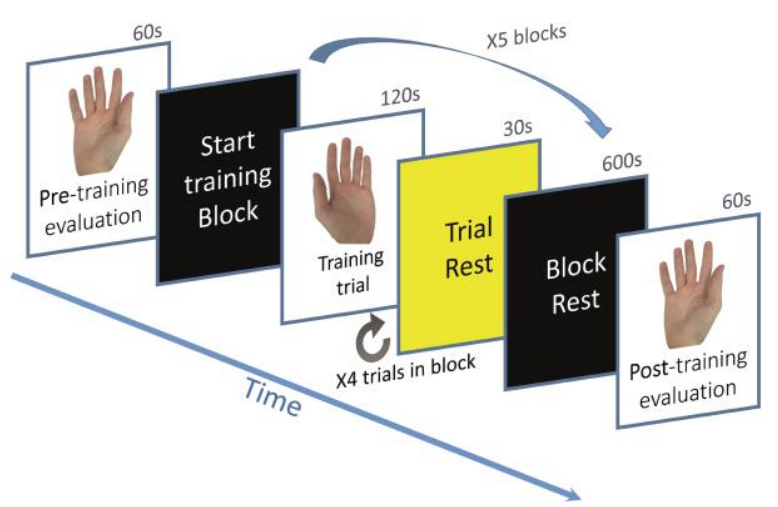

C

\section{experiment design}

\begin{tabular}{|c|c|c|c|c|c|c|}
$\begin{array}{c}\text { first } \\
\text { practice }\end{array}$ & $\begin{array}{c}\text { first } \\
\text { practice }\end{array}$ & $\begin{array}{c}\text { pre-baseline } \\
\text { clinical } \\
\text { evaluation }\end{array}$ & $\begin{array}{c}\text { baseline } \\
\text { training } \\
\text { (home) }\end{array}$ & $\begin{array}{c}\text { post-baseline } \\
\text { clinical } \\
\text { evaluation }\end{array}$ & rest & $\begin{array}{c}\text { retention } \\
\text { clinical } \\
\text { evaluation }\end{array}$ \\
\hline Day1 & Day2 & Day3 & Day3-12 & Day12 & Day13-18 & Day19
\end{tabular}

\begin{tabular}{|c|c|c|c|c|c|c|}
\hline $\begin{array}{l}\text { MRI scan } \\
\text { session } 1\end{array}$ & rest & $\begin{array}{l}\text { MRI scan } \\
\text { session 2+ } \\
\text { pre-VR clinical } \\
\text { evaluation }\end{array}$ & $\begin{array}{c}\mathrm{VR} \\
\text { training }\end{array}$ & $\mid \begin{array}{c}\text { MRI scan } \\
\text { session } 3+ \\
\text { post-VR clinical } \\
\text { evaluation }\end{array}$ & rest & $\begin{array}{l}\text { retention } \\
\text { clinical } \\
\text { evaluation }\end{array}$ \\
\hline Day19 & Day20-23 & Day24 & Day24-33 & Day33 & Day34-39 & Day40 \\
\hline
\end{tabular}

d

\section{fMRI stimulus}

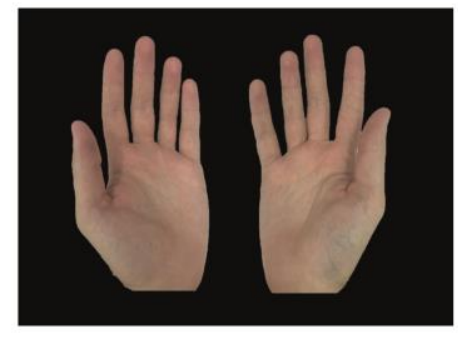

e $\quad$ fMRI session design

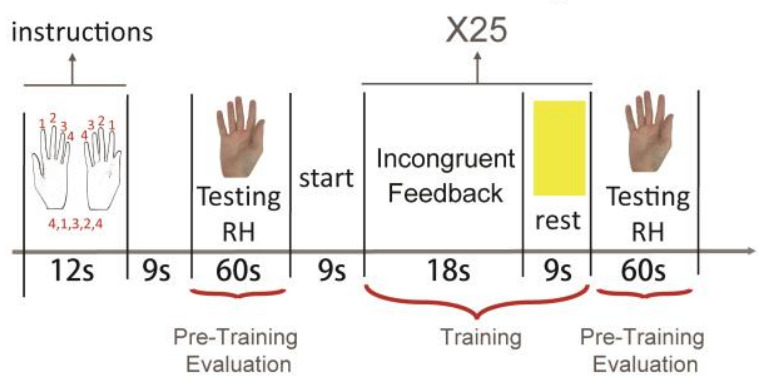

Fig. 1. Experiment design. (a) Experiment setup illustration. LA wore a VR headset with motion sensitive gloves (frontal view box) and received visual feedback of virtual hands (subject's view box). The VR devices allowed manipulation of online visual feedback. A camera mounted on the headset allowed embedding the virtual hands and patient's view inside a natural environment. The custom-built device was used with the virtual reality setup to produce yoked movement of the affected hand. (b) Schematic illustration of a single training day during the intervention period. The training days started with pre-training evaluation for 60 seconds in which LA conducted the sequence using his right hand. Then, 5 training blocks which included four 150-seconds trials in which LA conducted the sequence in our special setup. Finally, he performed a post-training evaluation similar to the first. (c) Schematic design of the complete experimental time-line. The experiment had two main parts - standard training (first two weeks; top row) and Intervention (last two weeks; bottom row). During the intervention, LA was trained in our special setup (see panel b for a single intervention day). (d) fMRI Training stimuli - picture of the virtual hands used as visual feedback during training in the fMRI sessions. (e) Schematic illustration of the time-line during fMRI training session in which LA performed the finger sequence with his left hand while receiving visual feedback of right virtual hand movement inside the fMRI scanner. 
blank screen to serve as a cue the resting period. The training stage consisted of 5 such training blocks (with overall training duration $=90$ minutes). After the training stage, LA's performance level in the affected hand was re-evaluated as previously for 60 seconds. During both pre- and post-training evaluation stages, LA was instructed to repeatedly execute the sequence as fast and as accurately as possible. The evaluation tests were performed while LA's hands were disconnected from the training apparatus. During the $9^{\text {th }}$ day of the intervention we encountered technical problems (computer motherboard failure) that precluded training during that day. The problem was resolved for the $10^{\text {th }}$ day of training. The clinical evaluations were performed in the first and last day of the intervention.LA also underwent another clinical test a week after the last day of the intervention in order to evaluate retention level. In addition, LA completed 3 sessions of fMRI scans in 3 different days: 4 days before the intervention period, the first day of intervention, and the last day of intervention (Fig. 1). The protocol was approved by the Ethics Committee of Tel-Aviv University and the Helsinki committee at Loewenstein Hospital.

\section{Behavior: motor sequence learning}

We evaluated changes in performance and kinematics of the affected hand following training with the non-affected hand. Evaluation of performance $(P)$ was based on the number of correctly performed 5-digit sequence within 60 seconds in the evaluation stages. Performance gains within each day were evaluated using equation (1) below:

$$
G=\frac{p_{\text {post_training }}-p_{\text {pre_training }}}{p_{\text {post_training }}+p_{\text {pre_training }}}
$$

Where $P_{\text {post_training }} / P_{\text {pre_training }}$ corresponds to the subject's performance in the post/pre training evaluation stages. Therefore, a positive $G$ index reflects improvement in performance. These gains were evaluated for each training day throughout the intervention period.

\section{Behavior: standardized clinical tests}

Clinical outcome measurements included a battery of commonly used standardized tests that focus on the speed and functional quality of unimanual movement. We measured performance of both left and right hands using the Fugl-Meyer test ${ }^{22}$, the Jebsen-Taylor test ${ }^{23}$ and the Box \& Blocks test ${ }^{24}$.

\section{Neuroimaging: task and stimuli}

Three identical $\mathrm{fMRI}$ sessions included a task and a resting-state session. The task session included two evaluation stages (congruent visual feedback) and a training stage (mirrored visual feedback). During the scans, LA lied supine with his arms to the side of his body and palms facing up. He could not see his hands during the scans. We recorded the finger movements using the same MR compatible gloves used during the intervention period that allowed yoking the movements of virtual hands presented on a screen to real hand movements. During the task scans, two virtual hands were presented on a screen with black background (see Fig. 1d), and LA viewed the screen through a tilted mirror mounted in front of his eyes. In each task stage, LA was presented with a similar instructionsslide as during the intervention period. In the evaluation stage, following the instructions, LA performed the sequence using his affected right hand $(\mathrm{RH})$ while receiving congruent visual feedback. Next, in 
the training stage, he physically trained on the sequence using his non-affected left hand (LH) while receiving real-time corresponding visual feedback of right virtual hand movement (mirrored visual feedback). Finally, he performed another evaluation stage with his affected hand. This functional run lasted 825 seconds. During the resting-state scan, LA was instructed to relax with his eyes closed for 387 seconds while whole-brain functional data was collected.

\section{Neuroimaging: $f M R I$ data acquisition and processing}

Blood oxygenation level dependent (BOLD) contrast was obtained on a 3T Siemens Prisma scanner with an 8 channel head coil located at the Strauss Computational Neuroimaging Center at TelAviv University, Tel-Aviv, Israel. An echo-planar imaging sequence was used to obtain the functional data (39 ascending interleaved axial slices, $4 \mathrm{~mm}$ thickness, slice gaps $=0 ; \mathrm{TR}=3000 \mathrm{~ms}$; flip angle $=$ $90^{\circ} ; \mathrm{TE}=30 \mathrm{~ms}$; in-plane resolution $=1.72 \times 1.72 \mathrm{~mm}$; matrix size $\left.=128 \times 128\right)$. In addition, anatomical reference was obtained by T1-weighted scan (voxel size $=1 \times 1 \times 1 \mathrm{~mm}$ ).

All fMRI data were processed using the BrainVoyager QX software (version 2.6, Brain Innovation, Maastricht, Netherlands; http://www.brainvoyager.com).

Prior to statistical analysis, a pre-processing procedure was performed on all functional images that included cubic spline slice-time correction, trilinear 3D motion correction, and high-pass filtering (above $0.006 \mathrm{~Hz}$ ). In addition, we assessed head movements and verified no scans contained head movement exceeding $2 \mathrm{~mm}$ in either direction. The 2D functional images were co-registered to the anatomical images. Functional data was spatially smoothed (Gaussian filter, FWHM $6 \mathrm{~mm}$ ) prior to statistical analysis.

\section{Neuroimaging: resting-state analysis}

To examine differences in functional connectivity between the pre- and post-intervention scans, we carried out whole-brain functional connectivity analysis during rest. For each scan, we parceled the brain to 111 cortical and sub-cortical regions according to the Oxford-Harvard atlas ${ }^{25}$. The functional time courses (129 volumes) were averaged across all voxels within each region. Pearson Correlation was used to determine the connectivity strength between each pair of regions, yielding three $111 \times 111$ symmetrical connectivity matrices (for two pre- and one post- training sessions).

\section{Neuroimaging: mirrored feedback analysis}

To detect regions exhibiting functional changes following training, we performed a general linear model (GLM) analysis on the fMRI data obtained during the mirrored task performance stages by

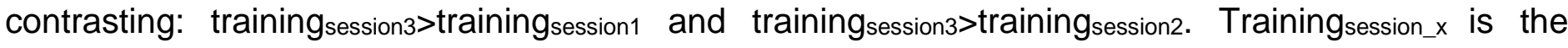
contrast activity during the mirrored task blocks in session $\mathrm{x} v \mathrm{vs}$. rest. The resulting maps were corrected by controlling the False Discovery Rate ${ }^{26}$ and thresholded at $\mathrm{q}(\mathrm{FDR})<0.05$, with a minimum cluster size of 50 voxels.

\section{Results}

\section{Behavior: motor sequence learning}

The affected right hand revealed a steady improvement in the motor sequence task during the intervention period (see Fig. 2a). A constant improvement is seen across the different days of intervention (slope of the linear regression $b=2.17 ; p=10^{-5}$ Pearson correlation $r=0.93$ ). A significant increase was found also within training days (averaged $G$ index across days $=0.09 ; p=6.6 \cdot 10^{-5}$ two- 
tailed unequal variance t-test compare to zero), with highest performance gains at the beginning and the middle of the intervention period (see Fig.2b).

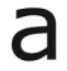

Behavioral Results

Task improvement

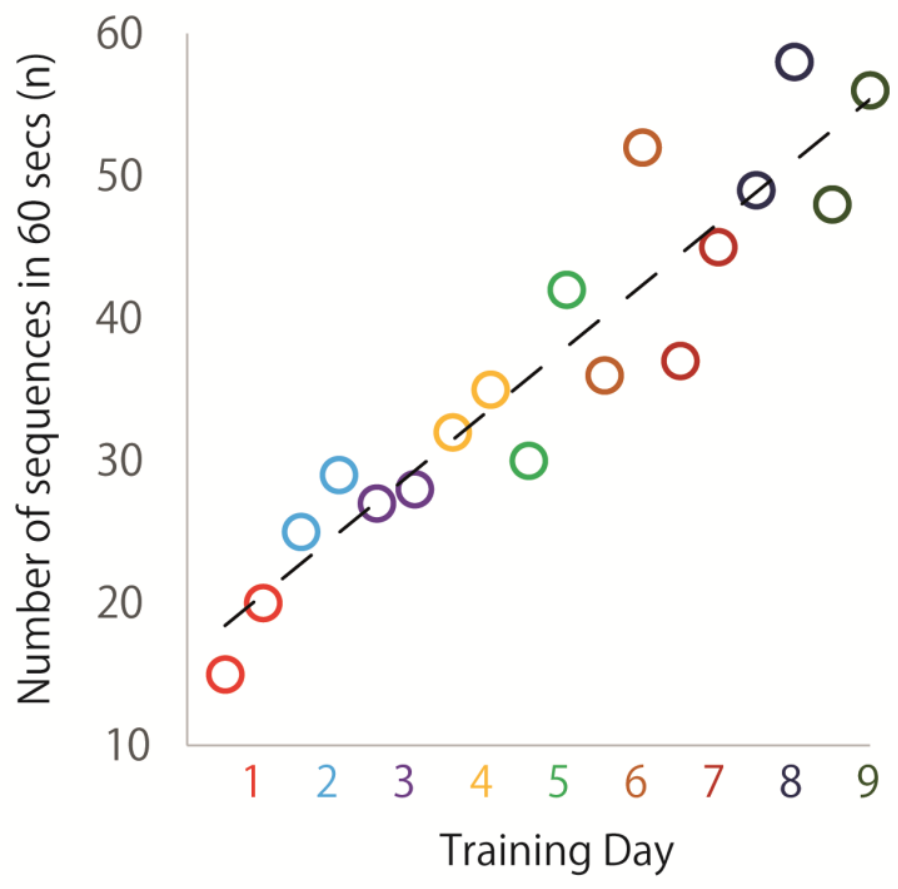

b

\section{Behavioral Results}

Daily performance gains

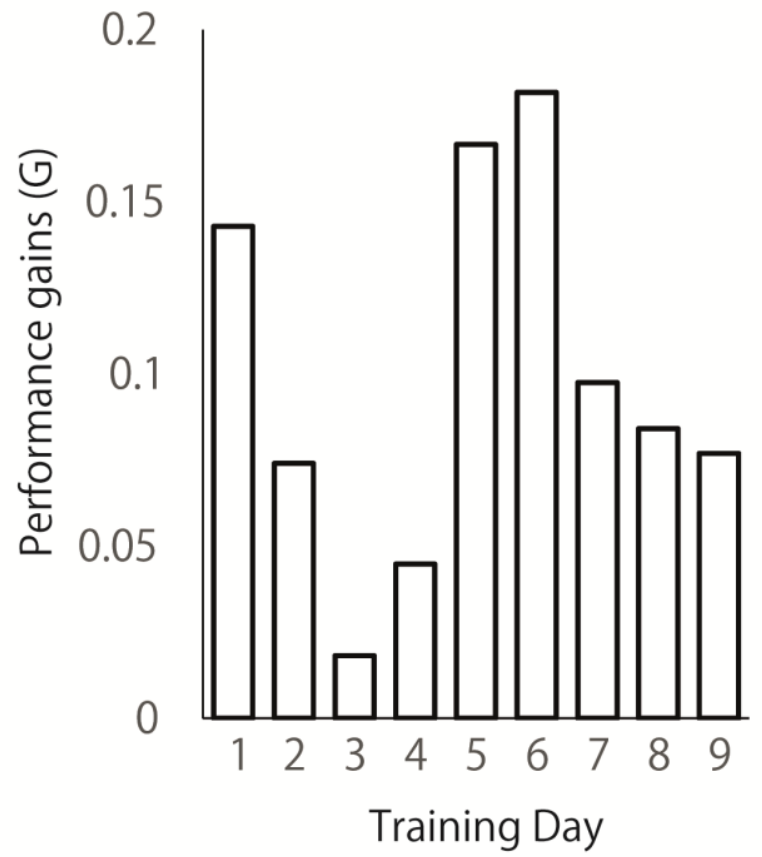

Fig. 2. Behavioral results - sequence task. (a) Scatter plot representing the number of sequences LA performed during the daily evaluation stages. Result indicate that training with the experimental setup during the intervention period yielded significant increase in the number of correct sequences performed by LA with the affected hand during the daily evaluation stages (each evaluation stage lasted 60 seconds). The first circle in each day is the pre-training evaluation stage and the second is the post-training evaluation (see Fig. 1b). Each color corresponds to a different day. (b) Bar graph representing the $G$ index in each day (reflecting LA's daily improvement from pre-evaluation stage to postevaluation stage; see Methods). Results indicate that improvement was highest during days $5 \& 6$ of the intervention period.

\section{Behavior: standardized clinical tests}

The Fugl-Meyer test for the right UL did not change following training and the score remained 53 out of 66 . In the Jebsen-Taylor test, we found differences in right hand functioning (measured as time to complete the task) between the 3 evaluation time-points, both in the standard training and the intervention periods (pre-training, post-training and retention tests; see Fig. 3 and Methods). In 3 tests, performance gains were higher during the intervention period compared with the standard training picking up beans with spoon (regression slope $=-0.88$ vs. -0.55 respectively; Fig. $3 d$ ), stacking checkers (-0.42 vs. -0.08 ; Fig. 3e), and moving full cans (-0.04 vs. 0.01 ; Fig. 3f). However, performance gains were better following standard training in flipping cards ( -0.5 vs. 0.04 ; Fig. 3a), flipping coins (0.25 vs. 0.01 ; Fig. $3 c$ ), and writing (-1.45 vs. -0.58 ; Fig. $3 g$ ). LA's progress in the 'moving empty cans' task was poor across the study ( 0.35 and 0.31 ; Fig. $3 b)$. In the Box \& Blocks test, LA exhibited high and consistent improvement across the study (2.5 vs. 4 ; Fig. $3 \mathrm{~h})$. 


\section{Behavioral Results}

\section{Clinical tests}

\section{a}
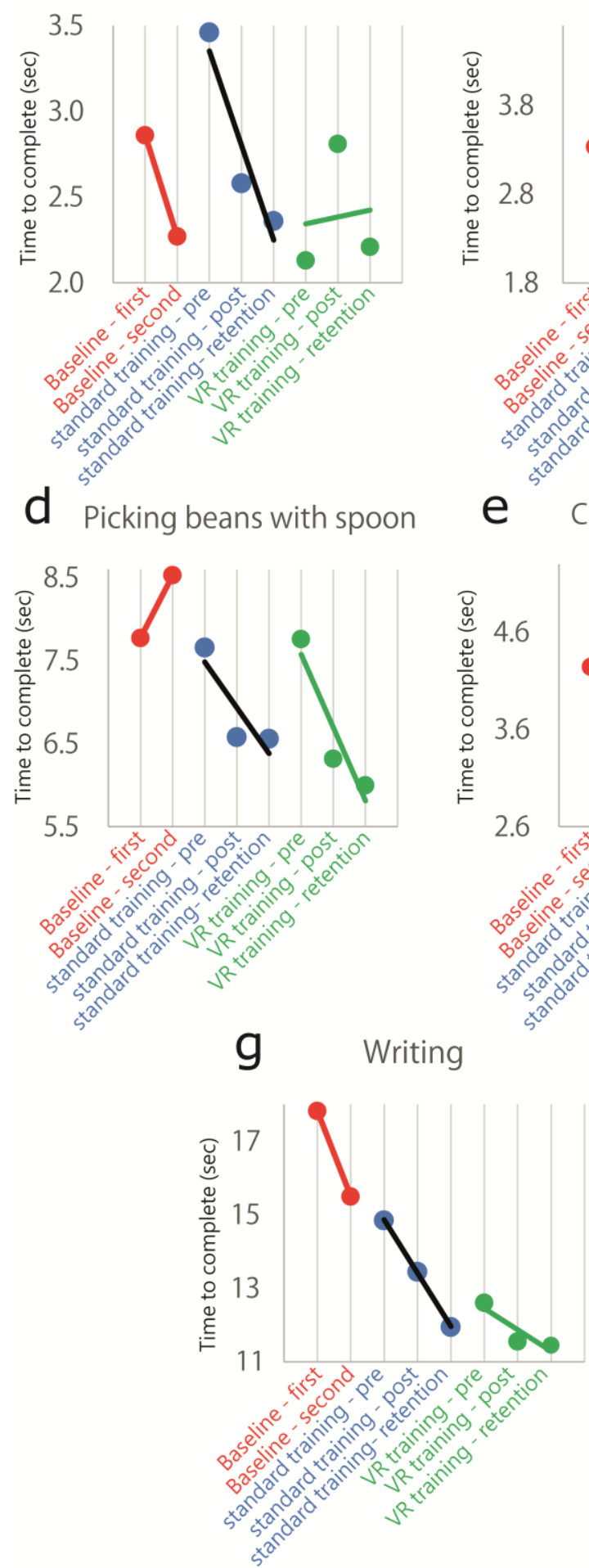

b Moving empty cans

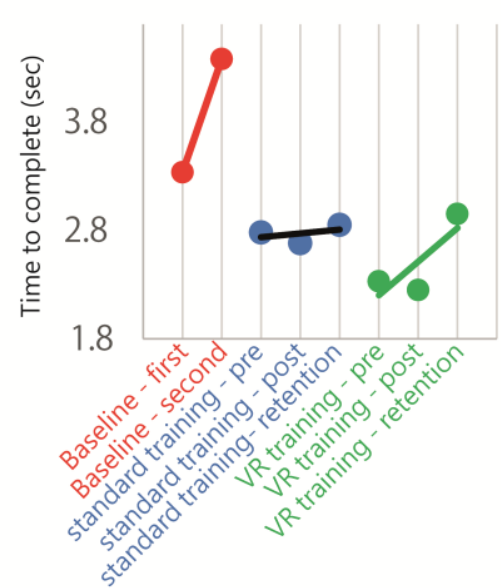

e Checkers stacking

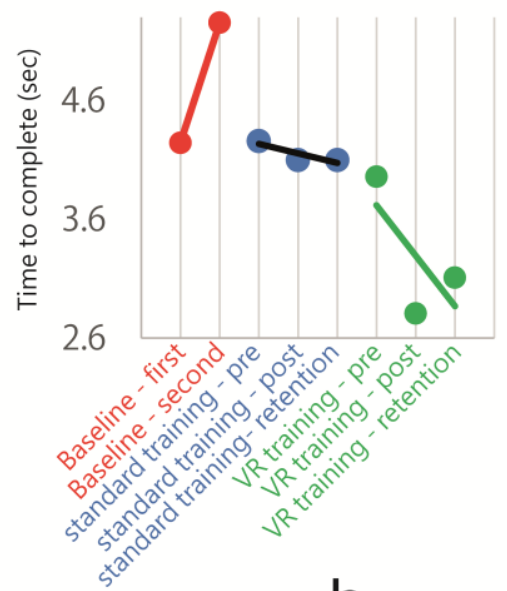

C Flipping coins
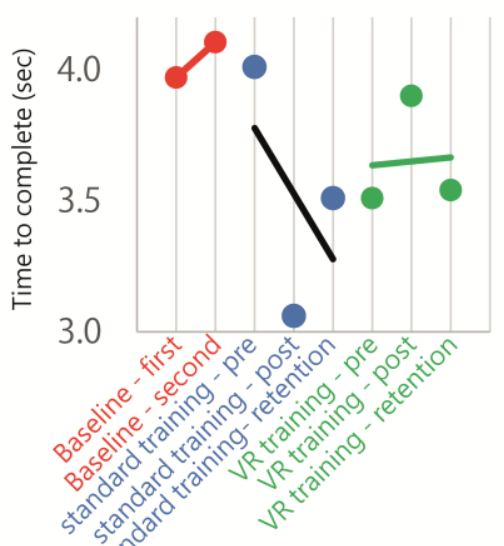

f Moving full cans

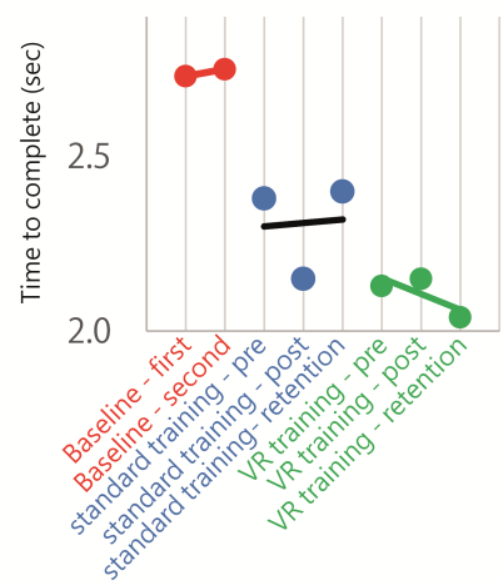

h Box \& Blocks

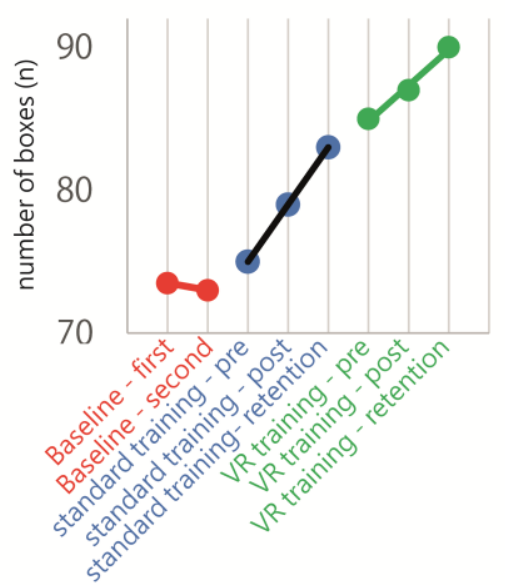

Fig. 3. Behavioral results - clinical tests. (a-g) Jebsen \& Taylor functional test results. Each panel shows results from different test. Plot reflects patient's performance in the first baseline tests (red; see experimental design in Fig. 1c), in the standard training (blue), and in intervention training (green). Patient exhibited changes both following the standard training and Intervention periods. The results were not conclusive across all the functional tests but there is a clear trend of global improvement (reduction in time to complete the task) throughout the experiment. (h) Box \& Blocks test results during baseline (red), standard training (blue) and intervention (green). LA consistently improved during the experiment. 
Fig. 4 depicts the activation contrast map of post-intervention scan (session 3) vs. preintervention scans (sessions $1 \&$ 2) during task performance blocks (see Methods). Several clusters in the pre-frontal cortex were more active after the intervention. These clusters are located mainly in the superior and medial frontal gyri. We also obtained a cluster in the medial occipital cortex, near the calcarine sulcus. Additionally, a small cluster located in the right primary motor and primary somatosensory cortices (contra-lateral to the moving hand, and ipsilateral to the affected hand) was more activated during the post-intervention session. In contrast, activation was lower in several regions that are key parts of the visual dorsal stream including superior parietal lobules (SPL), inferior parietal lobules (IPL) and the lateral occipital gyri in both right and left hemispheres. Results imply that our relatively short intervention period was sufficient to induce significant neural changes in frontal and visual pathways.

\section{Neuroimaging: resting state}

Resting-state functional connectivity revealed a global increase in the number of significantly connected regions in the post-intervention scan (560 regions) compared with the pre-intervention scans (239 regions, averaged across the two scans; see Fig. 4b). These results suggest that the intervention period strengthened functional connectivity within the brain network.

\section{Discussion}

The aim of the current study was to assess the clinical applicability of a novel treatment setup combining two principles: (a) cross education (CE) - obtaining performance gain in the affected upper $\operatorname{limb}(U L)$ indirectly, by intensive training of the non-affected UL; (b) Perceptual mirroring in two sensory systems - visual and proprioceptive, creating a manipulated perception of voluntary movement in the neurologically affected UL. In a recent study with healthy subjects, this setup showed high performance outcomes in the UL that was not physically trained ${ }^{8}$. Before embarking on a large scale RCT type clinical study, we decided to perform a detailed examination of the behavioral and brain activation dynamics related to training with the setup in a single-case design. Patient LA was selected because his marked unilateral bradykinesia and rapid fatigue prevented intensive direct training of the affected UL. LA revealed a clear learning curve in repeated performance of the motor sequence task by the affected right UL during the intervention period. These results point to stable and significant performance gains with the affected UL in the absence of its voluntary physical training. The improvement shown in sequence learning had a moderate correlation with the clinical tests.

Although much of the clinical research on rehabilitation of unilateral impairment of UL function focuses on voluntary physical practice with the affected UL, especially in motor impairment stemming from stroke ${ }^{27-29}$, this kind of movement-based intervention (e.g., constraint induced movement therapy, CIMT) is usually limited by the amount of volitional motion patients can actually produce ${ }^{30-32}$. Therefore, it is particularly important to examine alternative approaches, especially for the more severe cases in which direct training of the affected UL is not suitable. Our experimental intervention is such an alternative approach, motivated by knowledge gained from recent motor learning research in healthy subjects 7,33 . Our findings shows that this approach can have a useful clinical application in neurorehabilitation. It incorporates principles derived from mirror visual feedback (MVF) research showing the benefits of mirror therapy in a variety of clinical conditions ${ }^{34-37}$. We have recently reported two 

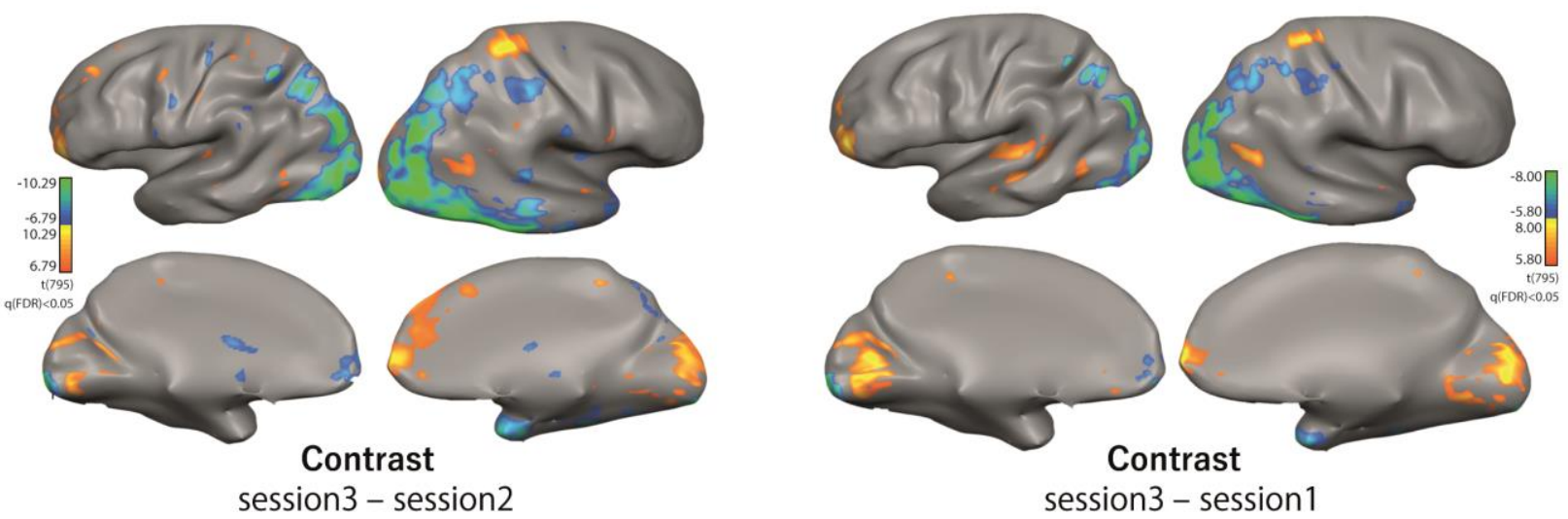

b

\section{Resting State Connectivity}
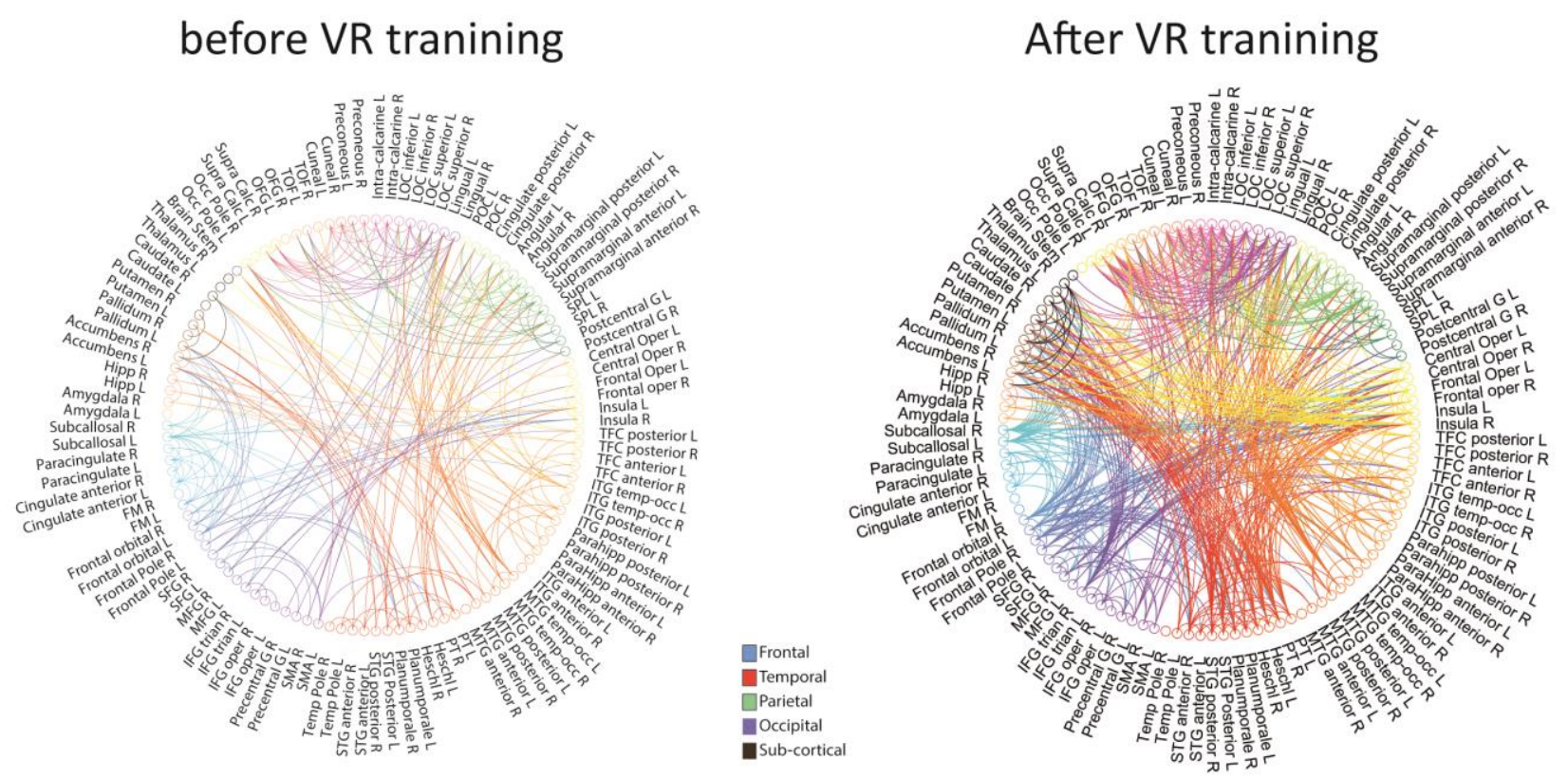

Fig. 4. fMRI results. (a) Activation maps displaying significant regions obtained from the GLM contrasts $(q(F D R)<0.05)$. Left panel shows the differences in activation during task performance between session 3 (end of experimental intervention) and session 2 (beginning of experimental intervention). Yellow regions represent activation that was significantly higher during session 3 compared to session 2 . In contrast, blue and green regions represent activation during task performance that was significantly higher during session 2. Similarly, the right panel shows differences in activation between session 3 (end of experimental intervention) and session 1 (5 days before the starting day of the experimental intervention). (b) Functional connectivity during resting-state. Brain regions (cortical and sub-cortical regions; color coded) were defined by the Oxford-Harvard atlas. Two regions are connected with a line if there is a significant correlation between their averaged time-courses during resting-state scan (See Methods). The two circles represent functional connections before and after the VR training. The left panel shows the connectivity between the regions before the VR training (the two pre-training sessions yielded similar connectivity maps, indicating that the fMRI dynamics did not change from one session to another before our VR intervention). The right panel shows the connectivity after the VR training. Results show significant increase in network connectivity after intervention. 
distinct electrophysiological counterparts of MVF implying (a) recruitment of mirror neurons and (b) attenuation of hemispheric asymmetry when the mirror reflection of the moving hand creates an illusory perception of movement in the other hand ${ }^{38}$. Here we extended the standard MVF approach by using visual feedback in a VR environment, which allows feedback to be controlled by software. Future studies using this environment can introduce various perturbations (in time and space, such as temporal delays or size changes in virtual hands ${ }^{39}$ ) to find optimal parameters for efficient rehabilitation.

At the neural level, regions in the pre-frontal cortex were more engaged during task performance after the intervention, while the visual dorsal stream was less engaged. The dorsal stream processes visual information needed for directed actions in space ${ }^{40}$, and plays a significant role in the actionobservation interaction ${ }^{41}$. In contrast, pre-frontal regions are engaged in action selection and reinforcement learning ${ }^{42,43}$. Pre-frontal regions have been found crucial in linking memory representations to goal-directed motor behavior and optimizing selection between competing responses ${ }^{44}$. Thus, we suggest that the intervention induces a processing shift during mirror training from the parietal-occipital network engaged in spatial processing to more frontal cognitive circuits. Importantly, we show that 10-days intervention strengthens interconnections in the brain. Further research with a higher number of patients and healthy controls is needed to understand the full effect of the intervention on connections strength.

To summarize, this case study demonstrates that cross education training with manipulated visual and proprioceptive feedback in a VR environment, can boost motor rehabilitation in patients with unilateral UL dysfunction. This is a single case and therefore the implications of the current results should be treated with caution. Unilateral motor impairment in neurology is a multi-faceted and multifactorial state, and benefits obtained in hemi-Parkinson's disease might not replicate with types of unilateral UL dysfunction involving mainly the pyramidal system. The strategy adopted here combines insights gained from basic motor neuroscience research and research conducted in the clinical setting, in the goal of developing a novel training regime for rehabilitation of patients with unilateral upper limb dysfunction. Although our results constitute a single case, they hold promise for future studies examining this line of treatment in additional clinical populations. 


\section{Acknowledgments}

The authors are thankful to patient LA for his cooperation in the study. We also thank O. Levy and Y. Siman-Tov from Rehabit-Tec System for providing access to the passive movement device. This study was supported by the I-CORE Program of the Planning and Budgeting Committee and the Israel Science Foundation (grant No. 51/11), and The Israel Science Foundation (grants No. 1771/13 and 2043/13) (R.M.); The Yosef Sagol Scholarship for Neuroscience Research, The Israeli Presidential Honorary Scholarship for Neuroscience Research, and the Sagol School of Neuroscience fellowship (O.O.). The funders had no role in study design, data collection and analysis, decision to publish, or preparation of the manuscript.

\section{Author contribution statement}

OO, LM, SFT, RM and NS designed the study. OO, EK, SK, RG, DR trained the patient and collected behavioral and fMRI data. SFT and LM collected clinical data under NS supervision. OO analyzed behavioral and neuroimaging data under RM supervision. OO, RM, and NS wrote the manuscript.

\section{Conflict of interest statement}

The authors declare that the research was conducted in the absence of any commercial or financial relationships that could be construed as a potential conflict of interest. 


\section{References}

1. Scripture E, Smith TL \& Brown EM (1894) On the education of muscular control and power. Stud Yale Psychol Lab 2:114-119.

2. Sainburg RL \& Wang J (2002) Interlimb transfer of visuomotor rotations: independence of direction and final position information. Experimental brain research 145: 437-447.

3. Anguera JA, Russell CA, Noll DC \& Seidler RD (2007) Neural correlates associated with intermanual transfer of sensorimotor adaptation. Brain research 1185: 136-151.

4. Hendy AM, Spittle M \& Kidgell DJ (2012) Cross education and immobilisation: mechanisms and implications for injury rehabilitation. Journal of science and medicine in sport 15: 94-101.

5. Farthing JP (2009) Cross-education of strength depends on limb dominance: implications for theory and application. Exercise and sport sciences reviews 37: 179-187.

6. Raghavan P, Krakauer JW \& Gordon AM (2006) Impaired anticipatory control of fingertip forces in patients with a pure motor or sensorimotor lacunar syndrome. Brain 129: 1415-1425.

7. Ossmy O \& Mukamel R (2016) Neural network underlying intermanual skill transfer in humans. Cell reports 17: 2891-2900.

8. Ossmy O \& Mukamel R (2017) Using Virtual Reality to Transfer Motor Skill Knowledge from One Hand to Another. $J$ Vis Exp.

9. Ossmy O \& Mukamel R (2018) Perception as a Route for Motor Skill Learning: Perspectives from Neuroscience. Neuroscience 382: 144-153.

10. Pelosin E, Bove M, Ruggeri P, Avanzino L \& Abbruzzese G (2013) Reduction of bradykinesia of finger movements by a single session of action observation in Parkinson disease. Neurorehabilitation and neural repair 27: 552-560.

11. Robles-García $V$ et al. (2013) Motor facilitation during real-time movement imitation in Parkinson's disease: a virtual reality study. Parkinsonism \& related disorders 19: 1123-1129.

12. Ghilardi M-F et al. (2000) Visual feedback has differential effects on reaching movements in Parkinson's and Alzheimer's disease. Brain research 876: 112-123.

13. Ertelt $D$ et al. (2007) Action observation has a positive impact on rehabilitation of motor deficits after stroke. Neuroimage 36: T164-T173.

14. Wong JD, Kistemaker DA, Chin A \& Gribble PL (2012) Can proprioceptive training improve motor learning? Journal of neurophysiology 108: 3313-3321.

15. Lotze M, Braun C, Birbaumer N, Anders S \& Cohen LG (2003) Motor learning elicited by voluntary drive. Brain 126: 866-872.

16. Hesse S, Schulte-Tigges G, Konrad M, Bardeleben A \& Werner C (2003) Robot-assisted arm trainer for the passive and active practice of bilateral forearm and wrist movements in hemiparetic subjects1. Archives of physical medicine and rehabilitation 84: 915-920.

17. Lindberg P, Schmitz C, Forssberg H, Engardt M \& Borg J (2004) Effects of passive-active movement training on upper limb motor function and cortical activation in chronic patients with stroke: a pilot study. Journal of Rehabilitation Medicine 36: 117-123.

18. Volpe BT, Krebs HI \& Hogan N (2001) Is robot-aided sensorimotor training in stroke rehabilitation a realistic option? Current opinion in neurology 14: 745-752.

19. Picelli A, Tamburin S, Passuello M, Waldner A \& Smania N (2014) Robot-assisted arm training in patients with Parkinson's disease: a pilot study. Journal of neuroengineering and rehabilitation 11:28. 20. Buchman AS, Goetz CG \& Klawans HL (1988) Hemiparkinsonism with hemiatrophy. Neurology 38: 527-527.

21. Giladi N et al. (1990) Hemiparkinsonism-hemiatrophy syndrome Clinical and neuroradiologic features. Neurology 40: 1731-1731. 
22. Fugl-Meyer AR, Jääskö L, Leyman I, Olsson S \& Steglind S (1975) The post-stroke hemiplegic patient. 1. a method for evaluation of physical performance. Scandinavian journal of rehabilitation medicine 7: 13-31.

23. Jebsen RH, Taylor N, Trieschmann R, Trotter MJ \& Howard LA (1969) An objective and standardized test of hand function. Archives of physical medicine and rehabilitation 50: 311-319.

24. Mathiowetz V, Volland G, Kashman N \& Weber K (1985) Adult norms for the Box and Block Test of manual dexterity. American Journal of Occupational Therapy 39: 386-391.

25. Smith SM et al. (2004) Advances in functional and structural MR image analysis and implementation as FSL. Neuroimage 23: S208-S219.

26. Benjamini $Y$ \& Hochberg $Y$ (1995) Controlling the false discovery rate: A practical and powerful approach to multiple testing. Journal of the Royal Statistical Society. Series B (Methodological) 57: 289300.

27. Grotta JC et al. (2004) Constraint-induced movement therapy. Stroke 35: 2699-2701.

28. Taub E, Uswatte G \& Pidikiti R (1999) Constraint-induced movement therapy: a new family of techniques with broad application to physical rehabilitation-a clinical review. Journal of rehabilitation research and development 36: 237-251.

29. Wolf SL et al. (2006) Effect of constraint-induced movement therapy on upper extremity function 3 to 9 months after stroke: the EXCITE randomized clinical trial. Jama 296: 2095-2104.

30. Hoare BJ, Wasiak J, Imms C \& Carey L. (2007).

31. Bard G \& Hirschberg G (1965) Recovery of voluntary motion in upper extremity following hemiplegia. Archives of physical medicine and rehabilitation 46: 567-572.

32. Skilbeck CE, Wade DT, Hewer RL \& Wood VA (1983) Recovery after stroke. Journal of Neurology, Neurosurgery \& Psychiatry 46:5-8.

33. Ossmy O \& Mukamel R (2016) Activity in superior parietal cortex during training by observation predicts asymmetric learning levels across hands. Scientific reports 6: 32133.

34. Ramachandran VS \& Altschuler EL (2009) The use of visual feedback, in particular mirror visual feedback, in restoring brain function. Brain 132: 1693-1710.

35. Rosén B \& Lundborg G (2005) Training with a mirror in rehabilitation of the hand. Scandinavian journal of plastic and reconstructive surgery and hand surgery 39: 104-108.

36. Sathian K, Greenspan AI \& Wolf SL (2000) Doing it with mirrors: a case study of a novel approach to neurorehabilitation. Neurorehabilitation and neural repair 14: 73-76.

37. Selles RW, Schreuders TA \& Stam HJ (2008) Mirror therapy in patients with causalgia (complex regional pain syndrome type II) following peripheral nerve injury: two cases. Journal of rehabilitation medicine 40:312-314.

38. Bartur $\mathrm{G}$ et al. (2015) Electrophysiological manifestations of mirror visual feedback during manual movement. Brain research 1606: 113-124.

39. Ossmy O \& Mukamel R (2017) Short term motor-skill acquisition improves with size of selfcontrolled virtual hands. PloS one 12: e0168520.

40. Goodale MA \& Milner AD (1992) Separate visual pathways for perception and action. Trends in neurosciences 15:20-25.

41. Lee J-H \& van Donkelaar P (2002) Dorsal and ventral visual stream contributions to perceptionaction interactions during pointing. Experimental Brain Research 143: 440-446.

42. Ridderinkhof KR, Van Den Wildenberg WP, Segalowitz SJ \& Carter CS (2004) Neurocognitive mechanisms of cognitive control: the role of prefrontal cortex in action selection, response inhibition, performance monitoring, and reward-based learning. Brain and cognition 56:129-140.

43. Rowe JB, Toni I, Josephs O, Frackowiak RS \& Passingham RE (2000) The prefrontal cortex: response selection or maintenance within working memory? Science 288: 1656-1660.

44. Hadland K, Rushworth M, Passingham R, Jahanshahi M \& Rothwell J (2001) Interference with performance of a response selection task that has no working memory component: an rTMS comparison of the dorsolateral prefrontal and medial frontal cortex. Journal of Cognitive Neuroscience 13: 1097-1108. 